\title{
Why Channel Hopping Makes Sense, even with IEEE802.15.4 OFDM at $2.4 \mathrm{GHz}$
}

\author{
Jonathan Muñoz ${ }^{1}$, Paul Muhlethaler ${ }^{1}$, Xavier Vilajosana ${ }^{2}$, Thomas Watteyne ${ }^{1}$ \\ ${ }^{1}$ Inria, Paris, France. \\ \{jonathan.munoz, paul.muhlethaler, thomas.watteyne\} einria.fr \\ ${ }^{2}$ Universitat Oberta de Catalunya, Barcelona, Catalonia, Spain. \\ xvilajosana@uoc.edu
}

\begin{abstract}
Since its 2015 revision, IEEE802.15.4 now supports the OFDM physical layer at $2.4 \mathrm{GHz}$, on top of the popular O-QPSK modulation. Chips capable of both O-QPSK and OFDM are currently available. The question now is whether OFDM is relevant for low-power wireless mesh networking; given its prevalence in $\mathbf{4 G}$ cellular, it could be a game-changer. We start by collecting a comprehensive connectivity dataset, $141,587,000$ data points, continuously over 21 days. We then show, in an entirely counter-intuitive manner, that OFDM alone is not enough to cope with external interference and multi-path fading, and we recommend combining OFDM with channel hopping at the link layer. In the presence of WiFi interference, we further recommend using OFDM option 1 MCS3, a fast $800 \mathrm{kbps}$ mode, which does not use frequency repetition, even though the latter is meant to increase frequency diversity.
\end{abstract}

Keywords-IoT Enabling Technologies, IoT Protocols and Standards, Ultra-low power IoT Technologies, Industrial IoT and Factory of Things, OFDM.

\section{INTRODUCTION}

Due to their ease of deployment and low cost, low power wireless mesh networks have become a connectivity solution for commercial, industrial, governmental, urban and personal applications.

The preferred standard for those wireless networks is IEEE802.15.4. It defines the physical (PHY) and Medium Access Control (MAC) layers. While the initial 2003 version of the standard only included O-QPSK (Offset Quadrature Phase-Shift Keying), the latest 2015 version has added OFDM (Orthogonal Frequency Division Multiplexing). This might be a game-changer for low-power wireless, as OFDM promised longer range and high data rates, provided, that is, OFDM is capable of coping with external interference and multi-path fading.

This paper contributes to analyzing the relevance of OFDM for low-power wireless applications. We base our analysis on a comprehensive connectivity dataset, $141,587,000$ points, collected continuously over 21 days. Entirely counter-intuitively, we show that OFDM is actually prone to external interference and multi-path fading in a very similar way to O-QPSK. We therefore recommend combining OFDM with channel hopping at the link layer. Because of external interference, we further recommend

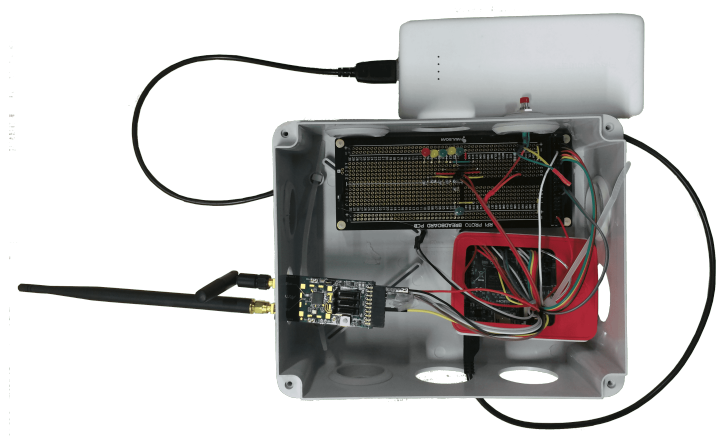

Figure 1: A test node consists of a Raspberry Pi driving an AT86RF215, capable of both O-PSK and OFDM at $2.4 \mathrm{GHz}$.

using fast communication (in particular OFDM option 1 MCS3), without frequency repetition, even when the latter appears to be, on paper, beneficial.

The remainder of this paper is organized as follows. Section II presents an overview of IEEE802.15.4 O-QPSK and OFDM. Section III enumerates the contributions of this paper. Section IV provides the details of the experimental setup. Section $\mathrm{V}$ analyzes the results and gives the lessons learned from this dataset. Finally, Section VI concludes this paper.

\section{OVERVIEW AND RELATED WORK}

The IEEE802.15.4-2015 standard [1] defines the PHY and MAC layer specifications for symmetric low data rate wireless mesh communication used by low-cost low-power devices. This standard can operate in the $2.4 \mathrm{GHz}$ unlicensed frequency band, which is what most deployments use. This technology has been deployed for industrial, urban, home and commercial applications. Most major chip vendors offer IEEE802.15.4-compliant radios at a low price, typically under 5 USD.

The first version of this standard appeared in 2003, and includes the O-QPSK PHY at $2.4 \mathrm{GHz}$. This PHY cuts the $2.4 \mathrm{GHz}$ frequency band into 16 channels. Each channel is $2 \mathrm{MHz}$ wide, adjacent channels are separated by $5 \mathrm{MHz}$. The data rate is $250 \mathrm{kbps}$; the maximum frame length is 
127 B. Direct Sequence Spread Spectrum (DSSS) is used to offer coding gain.

The latest 2015 version of this standard adds the OFDM (Orthogonal Frequency Division Multiplexing) PHY layer at 2.4 GHz. OFDM is a more recent technique than O-QPSK, borrowed from much more powerful networks such as $4 \mathrm{G}$ cellular. It is designed to combat multi-path fading at the PHY layer, something O-QPSK does not do. Fig. 2 shows how O-QPSK and OFDM channelize the same $2.4 \mathrm{GHz}$ frequency band.

OFDM has 4 ways of grouping sub-carriers to form an OFDM symbol; they are called "options". In this paper, we are interested in option 1, as it allows the highest data rate (and in particular data rates higher than O-QPSK). In OFDM option 1, each channel is composed of 104 subcarriers, each modulated independently. This means that a frame is actually composed of dozens of signals, all at a slightly different frequency within the channel. The idea is that multi-path fading and external interference only affect a portion of the sub-carriers. Smart inter-subcarrier coding compensates for that, resulting in a higher Packet Delivery Ratio (PDR) between neighbors.

Each sub-carrier is modulated. OFDM option 1 defines 2 sub-carrier modulations. It also defines the use of "frequency repetition", in which the same information is encoded onto multiple sub-carriers. The combination of modulation and level of frequency repetition is called Modulation and Coding Scheme (MCS). In this paper, we look at the MCS settings which yield the highest and lowest data rates: MCS0 and MCS3. Table I summarizes the MCS settings explored in this paper.

Wireless is unreliable by its very nature. In low-power wireless, the two phenomena that most impact the reliability of wireless links are external interference and multi-path fading.

The effect of external interferences on IEEE802.15.4 is well studied. Khaleel et al. [2] study the interference between IEEE802.15.4 and IEEE802.11b WiFi. By using the RSSI indicator, they sense the frequency spectrum under different $\mathrm{WiFi}$ data rate conditions. They conclude that, under the range of a WiFi connection of $3 \mathrm{Mbit} / \mathrm{s}$ and operating at the same frequency, an IEEE802.15.4 device is not capable of successfully accessing the medium up to $90 \%$ of the time.

Guo et al. [3] analyze how different Interference Sources increment the Packet Error Rate for IEEE80.15.4 radio links. With combinations of Interference Source (None, WiFi, Bluetooth and Microwave), distance between TX and RX, distance between IS and RX, position of IS in relation to TXRX and IEEE802.15.4 channel; the authors observed that $\mathrm{WiFi}$ and microwave ovens can degrade the quality of the radio links, causing a $25 \%$ PER depending of the distances between TX, RX, IS and as well as the chosen channel.

In order to better understand the impact WiFi net- works have on IEEE802.15.4 networks, Watteyne et al. [4] record the connectivity between 350 nodes on the IoT-lab testbed [5], located in an office building with WiFi networks around. Nodes communicate on the 16 frequencies defined in the O-QPSK PHY. The authors show that WiFi beacons (including when the WiFi network sits idle) hamper the connectivity on a considerable number of IEEE802.15.4 links, causing a drop in their PDR from $90 \%$ to $70-80 \%$.

Watteyne et al. [6] observe how multi-path fading affects wireless communication. They have a node transmit frames from a robotic arm that changes its position by steps of $1 \mathrm{~cm}$ inside a $20 \mathrm{~cm} \times 35 \mathrm{~cm}$ area. In total, the arm goes over to 735 positions, with the transmitter node sending 1,000 frames of $29 \mathrm{~B}$ at each position to a receiver node located $5 \mathrm{~m}$ away. This experiment is performed 16 times, one for each IEEE802.15.4 O-QPSK frequency at $2.4 \mathrm{GHz}$. The results show that moving the transmitter node by $3 \mathrm{~cm}$ can make the PDR of the wireless link to go from $100 \%$ to $0 \%$.

Time Synchronized Channel Hopping (TSCH) deals with external interference and multi-path fading at the Medium Access Control (MAC) layer. When two neighbor nodes exchange frames, they send subsequent frames at different frequencies, resulting in channel hopping. The idea is that, if external interference or multi-path fading causes the transmission of a frame to fail, the retransmission happens at a different frequency, and therefore has a higher chance of succeeding than if retransmitted on the same frequency.

The IETF 6TiSCH working group standardizes how to combine TSCH with IPv6. Watteyne et al. [7] show the performance on a commercial TSCH solution, SmartMesh IP, providing over $99.999 \%$ end-to-end reliability with over 10 years of battery lifetime. TSCH and 6TiSCH networks are considered the future of low power wireless [8].

Similar to TSCH, OFDM is a technique that copes with external interference and multi-path fading. Yet, unlike $\mathrm{TSCH}$, this happens at the PHY layer.

Lee et al. [9] combine a 32-bit micro-controller with an FPGA and a radio frequency ASIC to create a smart home system using IEEE802.15.4 FSK and OFDM. The authors are able to consult the home's water and electricity consumption remotely over the Internet.

In our previous publication [10], we conduct a series of experiments using one fixed transmitter node and 3 receiver nodes. The nodes consist of a Raspberry Pi, a battery pack and an ATREB215-Xplained pro board featuring an AT86RF215 radio chip implementing IEEE802.15.4 OQPSK, FSK and OFDM at sub-GHz and $2.4 \mathrm{GHz}$. All nodes are mounted on $1.8 \mathrm{~m}$ poles. In that paper, we explore 4 PHYs: O-QPSK at $2.4 \mathrm{GHz}$, OFDM option 1 MCS2 at sub$\mathrm{GHz}$, OFDM option $1 \mathrm{MCS} 3$ at sub-GHz, and OFDM option 2 MCS5 at sub-GHz. For each PHY, the transmitter sends 100127 B and 2047 B frames over all available frequencies in each PHY. We show that the IEEE802.15.4 OFDM PHYs 


\begin{tabular}{|l|l|l|l|l|l|l|l|}
\hline PHY & data rate & sensitivity & condition & TX current $(+8 \mathrm{dBm})$ & RX current & sub-carrier modulation & freq. rep. \\
\hline O-QPSK & $250 \mathrm{kbps}$ & $-104 \mathrm{dBm}$ & $\begin{array}{l}\text { PSDU length 20 B } \\
\text { PER }<1 \%\end{array}$ & $63.05 \mathrm{~mA}$ & $31.85 \mathrm{~mA}$ & N.A. & N.A. \\
\hline $\begin{array}{l}\text { OFDM } \\
\text { Option 1 MCS0 }\end{array}$ & $100 \mathrm{kbps}$ & $-112 \mathrm{dBm}$ & $\begin{array}{l}\text { PSDU length 250 B } \\
\text { PER }<10 \%\end{array}$ & $67.25 \mathrm{~mA}$ & $30.65 \mathrm{~mA}$ & BPSK rate $1 / 2$ & $4 \times$ \\
\hline $\begin{array}{l}\text { OFDM } \\
\text { Option 1 MCS3 }\end{array}$ & $800 \mathrm{kbps}$ & $-105 \mathrm{dBm}$ & $\begin{array}{l}\text { PSDU length 250 B } \\
\text { PER }<10 \%\end{array}$ & $67.15 \mathrm{~mA}$ & $30.65 \mathrm{~mA}$ & QPSK rate $1 / 2$ & none \\
\hline
\end{tabular}

Table I: Radio characteristics and details of the PHY explored in this paper.

in sub-GHz are suitable for Smart Building applications due to their higher PDR and lower power consumption in comparison with the well-known O-QPSK PHY.

One major difference with the current paper is that OFDM is evaluated at sub-GHz; this changes everything from a wireless point of view (interference, multi-path, etc). None of the lessons learnt from the current paper could have been possible from the data gathered for [10]. That being said, we refer the interested reader to [10] for an in-depth overview of OFDM, albeit at sub-GHz.

Surprisingly, little related work exists on evaluating the usefulness of OFDM in low-power wireless, even less at $2.4 \mathrm{GHz}$. This paper contributes to filling that void.

Specifically, the goal of this paper is to answer the following questions. Is OFDM a valid alternative for lowpower wireless? How does OFDM compare to O-QPSK in the IEEE802.15.4 context? How should OFDM be used at $2.4 \mathrm{GHz}$ ?

\section{CONTRibutions}

This paper focuses on IEEE802.15.4 OFDM option 1 (both MCS0 and MCS3), and O-QPSK, at $2.4 \mathrm{GHz}$. Its contributions are 4-fold:

1) We define a methodology and provide tools to continuously evaluate the PDR of a wireless link using off-the-shelf hardware.

2) We collect a connectivity dataset which contains $141,587,000$ points; to the best of our knowledge the most comprehensive OFDM/O-QPSK dataset at $2.4 \mathrm{GHz}$ to date.

3) In an entirely counter-intuitive manner, we show that channel hopping (at the MAC layer) is needed to achieve reliable connectivity, even when using IEEE802.15.4 OFDM at 2.4 GHz.

4) In a similarly counter-intuitive manner, we recommend not using frequency repetition to cope with external WiFi interference.

\section{EXPERIMENTAL SETUP}

This section provides all the necessary information for the reader to reproduce the experiments. This study is carried out by successive experiments using 4 nodes: one transmitter (TX) and three receivers (RX). In an experiment, the four nodes configure their radios with the three PHYs described in III. In O-QPSK, the nodes tune their radios to the

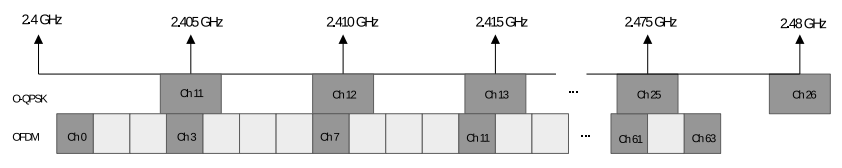

Figure 2: The O-QPSK PHY of IEEE802.15.4-2015 divides the $2.4 \mathrm{GHz}$ band into 16 channels, each $2 \mathrm{MHz}$ wide, separated by $5 \mathrm{MHz}$. The OFDM option 1 PHY divides the band into 64 channels, $1.094 \mathrm{MHz}$ wide, separated by $1.2 \mathrm{MHz}$. Greyed-out channels are the ones used in our experiments.

16 channels available in the $2.4 \mathrm{GHz}$ frequency band. In OFDM, we switch between 17 channels, a subset of the 64 available (see rationale in Section IV-B).

\section{A. Hardware}

A node consists of a Raspberry Pi 3 (rPi) model B and an ATREB215-XPRO radio board (featuring the AT86RF215 chip capable of both O-QPSK and OFDM), and a $2 \mathrm{dBi}$ omni-directional antenna. The rPi drives the radio board through an SPI bus. The electronic devices, connectors and cables are placed inside a plastic box. The antennas are fixed outside the box. Fig. 1 shows a picture of the node. Table I shows the performance of the radio.

\section{B. Software}

A Linux Debian distribution is running on the $\mathrm{rPi}$ of each node. Nodes are synchronized over NTP. A number of scripts drive the experiment ${ }^{1}$. These scripts configure the radios with a specific PHY, frequency, and for enough time to allow the TX node to send 1,000 $127 \mathrm{~B}$ frames. TX power is $+8 \mathrm{dBm}$; the spacing between frames is $20 \mathrm{~ms}$. The experiment runs continuously: the nodes loop through all PHY settings, each time sending 1,000 frames.

Because looping over 64 channels for OFDM would be too time consuming, they loop over the subset of 17 channels listed in Table II, which are chosen to almost match the frequencies used in O-QPSK. In total, 50 channels are tested: 16 in O-QPSK, 17 in both OFDM option 1 MCS0 and MCS3.

\footnotetext{
${ }^{1}$ The software used for these experiments is available under an opensource license at https://github.com/openwsn-berkeley/range_test/releases/ $\operatorname{tag} /$ REL-3.0.0.
} 


\begin{tabular}{|l|l|}
\hline $\begin{array}{l}\text { center } \\
\text { frequency }\end{array}$ & $\begin{array}{l}\text { O-QPSK } \\
\text { channel }\end{array}$ \\
\hline $2.400 \mathrm{GHz}$ & \\
\hline $2.405 \mathrm{GHz}$ & 11 \\
\hline $2.410 \mathrm{GHz}$ & 12 \\
\hline $2.415 \mathrm{GHz}$ & 13 \\
\hline $2.420 \mathrm{GHz}$ & 14 \\
\hline $2.425 \mathrm{GHz}$ & 15 \\
\hline $2.430 \mathrm{GHz}$ & 16 \\
\hline $2.435 \mathrm{GHz}$ & 17 \\
\hline $2.440 \mathrm{GHz}$ & 18 \\
\hline $2.445 \mathrm{GHz}$ & 19 \\
\hline $2.450 \mathrm{GHz}$ & 20 \\
\hline $2.455 \mathrm{GHz}$ & 21 \\
\hline $2.460 \mathrm{GHz}$ & 22 \\
\hline $2.465 \mathrm{GHz}$ & 23 \\
\hline $2.470 \mathrm{GHz}$ & 24 \\
\hline $2.475 \mathrm{GHz}$ & 25 \\
\hline $2.480 \mathrm{GHz}$ & 26 \\
\hline
\end{tabular}

\begin{tabular}{|l|l|}
\hline $\begin{array}{l}\text { OFDM } \\
\text { channel }\end{array}$ & $\begin{array}{l}\text { center } \\
\text { frequency }\end{array}$ \\
\hline 0 & $2.4012 \mathrm{GHz}$ \\
\hline 3 & $2.4048 \mathrm{GHz}$ \\
\hline 7 & $2.4096 \mathrm{GHz}$ \\
\hline 11 & $2.4144 \mathrm{GHz}$ \\
\hline 16 & $2.4204 \mathrm{GHz}$ \\
\hline 20 & $2.4252 \mathrm{GHz}$ \\
\hline 24 & $2.4300 \mathrm{GHz}$ \\
\hline 28 & $2.4348 \mathrm{GHz}$ \\
\hline 32 & $2.4396 \mathrm{GHz}$ \\
\hline 36 & $2.4444 \mathrm{GHz}$ \\
\hline 41 & $2.4504 \mathrm{GHz}$ \\
\hline 45 & $2.4552 \mathrm{GHz}$ \\
\hline 49 & $2.4600 \mathrm{GHz}$ \\
\hline 53 & $2.4648 \mathrm{GHz}$ \\
\hline 57 & $2.4696 \mathrm{GHz}$ \\
\hline 61 & $2.4744 \mathrm{GHz}$ \\
\hline 63 & $2.4768 \mathrm{GHz}$ \\
\hline
\end{tabular}

Table II: Center frequencies of the channels used for IEEE802.15.4 O-QPSK and OFDM

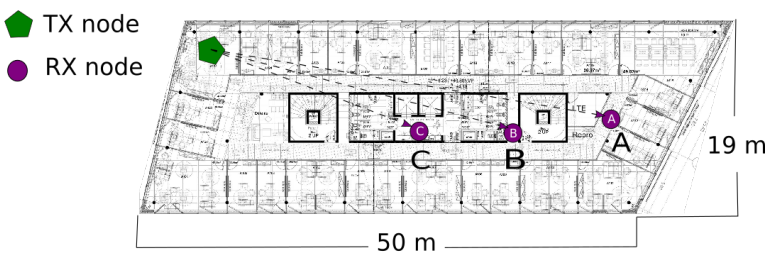

Figure 3: The location of the nodes in the Inria-Paris building during the 21-day experiment.

For each frame it receives, the RX node logs the PHY and channel used, the sequence number in the received frame, the RSSI value and whether the FCS is correct. Since 1,000 frames are sent, we can compute the PDR for each PHY/channel combination, and see its evolution over time.

\section{Deployment Environment}

We conduct the experiments within the Inria-Paris building in France. Fig. 3 shows the location of the nodes. The floor is covered with carpet, the ceiling is metallic, with two concrete staircases and two elevator shafts in the center of the floor. Divisions between offices are made of glass and prefabricated walls. External walls are made of concrete with glass windows.

The nodes are installed on $1.8 \mathrm{~m} \mathrm{PVC}$ poles. Experiments were conducted for 21 continuous days, with people around and WiFi being actively used during business hours.

\section{EXPERIMENTAL RESULTS}

We collected a dataset of $141,587,000$ atomic measurements. This section lists the lessons learnt from analyzing this dataset.

\begin{tabular}{|l|l|l|l|}
\hline PHY & Node A & Node B & Node C \\
\hline $\begin{array}{l}\text { O-QPSK } \\
(250 \mathrm{kbps})\end{array}$ & $82 \%$ & $61 \%$ & $83 \%$ \\
\hline $\begin{array}{l}\text { OFDM Option 1 MCS0 } \\
(100 \mathrm{kbps})\end{array}$ & $85 \%$ & $76 \%$ & $92 \%$ \\
\hline $\begin{array}{l}\text { OFDM Option 1 MCS3 } \\
(800 \mathrm{kbps})\end{array}$ & $92 \%$ & $77 \%$ & $93 \%$ \\
\hline
\end{tabular}

Table III: PDR for each node, averaged over time and over all channels.

\section{A. On Power Consumption}

Table I shows the current consumption of the radio for the 3 PHYs. The most energy-hungry operation a node performs is to have its radio on. An advanced MAC layer such as TSCH turns the radio off most of the time; a radio duty cycle below $1 \%$ is commonplace. The results about current draw from our previous publication [10] hold in this analysis; we hence refer the interested reader to that publication.

\section{B. On the PDR over the $2.4 \mathrm{GHz}$ frequency band}

Table III shows the PDR of the three links for each PHY layer, averaged over time and over all channels. While it is impossible to draw any general conclusion about data gathered over just 3 links, we can make two interesting observations. First, OFDM consistently yields a better PDR than O-QPSK. Second, MCS3 yields a better PDR than MCS0, even when it offers a much higher data rate and uses no frequency repetition. This will be explained in Section V-F.

The average PDR does not give us nearly as many insights as Fig. 4, which plots the PDR of each link evolving during the 21 days of the experiment, for each channel. Fig. 4 is for node $\mathrm{B}$, equivalent lessons learnt appear for the other nodes. Drawing a subplot for each channel allows us to "see" the effect of external interference and multi-path fading on a channel-by-channel basis. For easier readability, each subplot is highlighted in green when PDR $>50 \%$. This section makes a number of qualitative observations which are analyzed in greater detail in subsequent sections.

Fig. 4 shows that there is no single frequency that is "good" all the time. The PDR on each frequency is dynamic, as a result of changes in the environment. One can clearly see week-end/weekday and day/night transitions.

These dynamics in PDR are caused by multi-path fading and external interference. It is entirely expected - and well documented - in the IEEE802.15.4 O-QPSK case. In an entirely counter-intuitive manner, they are also equally present in OFDM. OFDM was designed to combat multi-path fading and external interference at the PHY layer, by encoding data on dozens of frequencies. One would expect the resulting frequency diversity at the PHY layer to cause the per-channel PDR to be very stable. The reason we $d o$ see PDR dynamics in OFDM is that the channels are only $1.094 \mathrm{MHz}$ wide: 


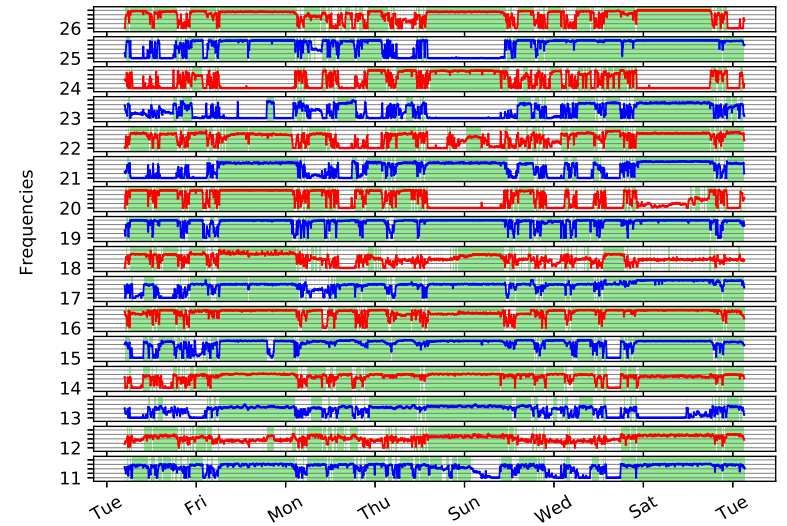

(a) O-QPSK (250 kbps)

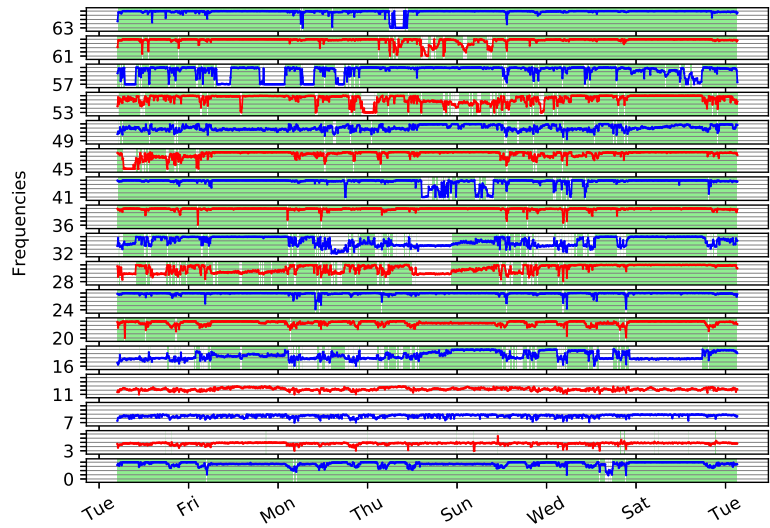

(b) OFDM option 1 mcs 0 (100 kbps)

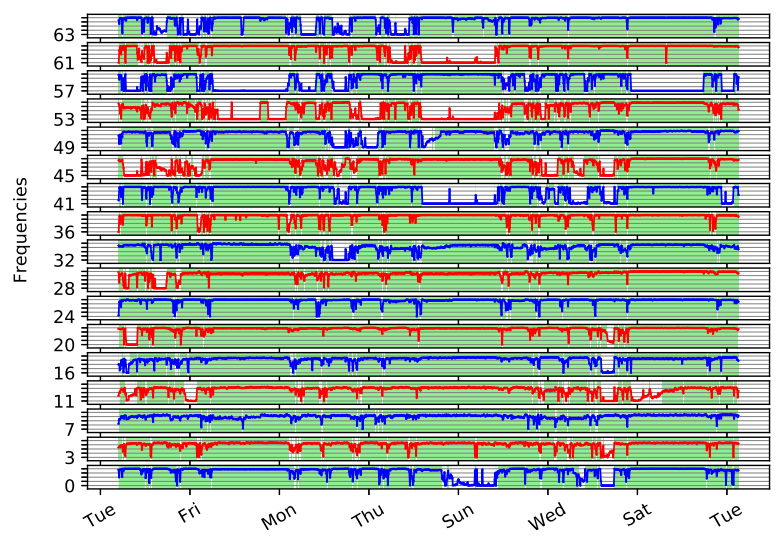

(c) OFDM option $1 \mathrm{mcs} 3$ (800 kbps)

Figure 4: PDR per PHY channel for node B during 21 days. Areas in green indicate PDR greater than 50\%.

multi-path fading and external interference affect all subcarriers at the same time. As a result, OFDM alone does not provide a sufficient mechanism in IEEE802.15.4 at $2.4 \mathrm{GHz}$ to combat external interference and multi-path fading.

\section{Impact of Nearby WiFi}

Fig. 4(b) shows a constantly bad link for channels 3, 7, 11. This can be attributed to external interference. To verify this, we use the iwlist Linux utility to list the RSSI and frequency of the WiFi signals received by node B, see Fig. 5 . WiFi activity is centered on WiFi channels $1(2.412 \mathrm{GHz}), 6$ $(2.437 \mathrm{GHz})$ and $11(2.462 \mathrm{GHz})$. Node B receives $21 \mathrm{WiFi}$ signals; the highest noise level is in the $2.404-2.414 \mathrm{GHz}$ band, the same as channels 3, 7, 11 in Fig. 4(b).

Section V-F further details why the same does not happen to MCS3.

\section{Quantifying Multi-path Fading in OFDM}

Multi-path fading causes some frequencies to exhibit a PDR above 50\%, others below. The experiment is conducted

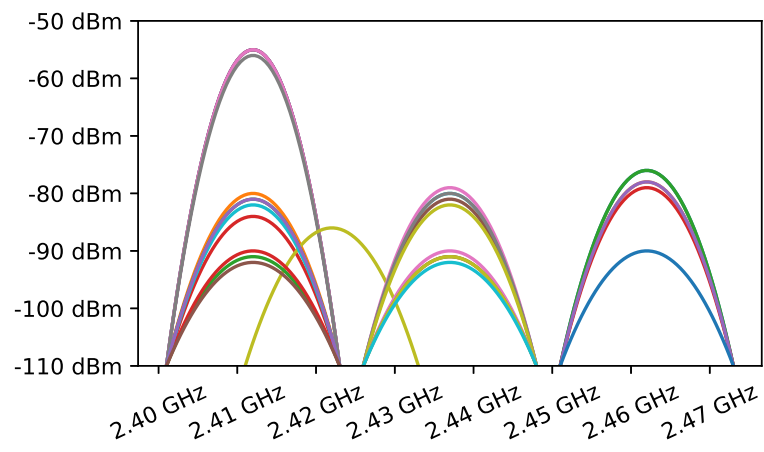

Figure 5: Strengths of the WiFi signals received at node B.

in cycles of approximately $30 \mathrm{~min}$, during which the TX node sends 1,000 frames on each PHY and channel. This means that, every $30 \mathrm{~min}$, we can compute the PDR of each link, on each channel, for each PHY.

Fig. 6 plots the number of channels which exhibit a PDR 


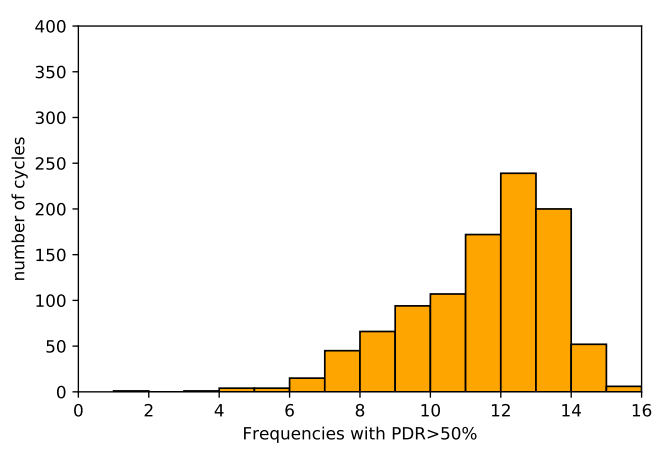

(a) O-QPSK (250 kbps)

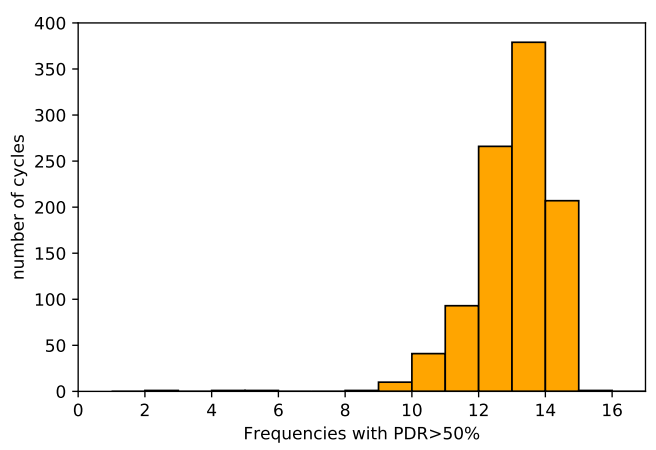

(b) OFDM option 1 MCS0 (100 kbps)

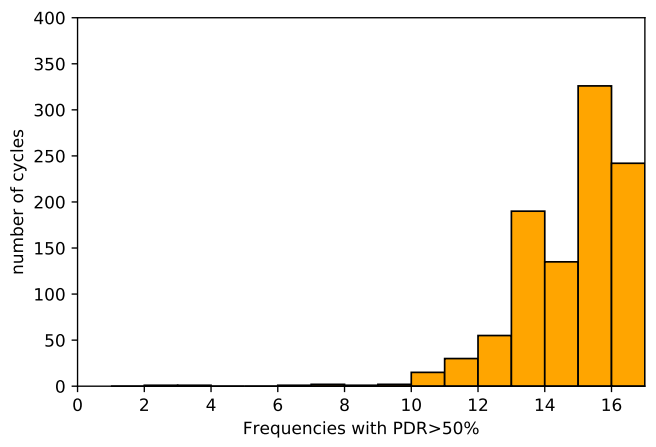

(c) OFDM option $1 \mathrm{MCS} 3$ (800 kbps)

Figure 6: Number of frequencies with PDR $>50 \%$, for node B.

above $50 \%$. In the absence of external interference and multi-path fading, either all channels would be good, or bad. That is, there would be a single bar at the maximum number of channels.

Rather, we see that, for O-QPSK, in most of the cases 13 channels are "good", the remaining 3 being affected by multi-path fading or interference. This is a simple way of "seeing" those effects, and is entirely expected for OQPSK. What is counter-intuitive is that OFDM is affected by multi-path fading and external interference in a way similar to O-QPSK.

\section{E. Coherence Bandwidth}

When node $A$ sends a frame to node $B$ on frequency $f_{1}$, and that transmission fails because of multi-path fading, a valid approach is to re-transmit on a different frequency $f_{2}$. We are interested in understanding how far $f_{2}$ should be from $f_{1}$ to offer enough frequency diversity. We call "coherence bandwidth" the difference $\left|f_{2}-f_{1}\right|$ which is sufficient for a link to transition from "bad" $(\mathrm{PDR}<50 \%)$ to "good" (PDR $>50 \%)$.

We explore this experimentally in our dataset: we find a frequency with PDR $<50 \%$, and see how big a frequency shift is needed to result in PDR $>50 \%$. Fig. 7 shows the result. When using O-QPSK (fig. 7(a)) and OFDM option 1
MCS3 (fig. 7(c)), a frequency shift of $10 \mathrm{MHz}$ is sufficient $60 \%$ of the time. For OFDM option 1 MCS0, a frequency shift of $10 \mathrm{MHz}$ is sufficient $40 \%$ of the time, $80 \%$ of the time with a shift of $20 \mathrm{MHz}$.

\section{F. Pros and Cons of Frequency Repetition}

Our previous publication [10] shows how frequency repetition improves the PDR of links at sub-GHz frequencies. Higher levels of frequency repetition should result in a higher PDR. This is not what we see when comparing MCS3 (no repetition) to MCSO $(4 \times$ repetition).

The reason is that frequency repetition increases the time on air of a frame, making it more prone to collisions with other radio signals. As seen in Fig. 5, there are WiFi networks in the proximity of node B, each of which emits beacons at least every $100 \mathrm{~ms}$, even when the WiFi network sits idle. A 127 B frame takes with OFDM option 1 MCS0 (100 kbps) roughly $10 \mathrm{~ms}$ to be transmitted, only $1.27 \mathrm{~ms}$ with MCS3 ( $800 \mathrm{kbps}$ ). Frequency Repetition is useful only if there is no strong external interference.

\section{CONClusions AND Future Work}

This paper investigates the usefulness of IEEE802.15.4 OFDM at $2.4 \mathrm{GHz}$, how it compares to O-QPSK, and how 


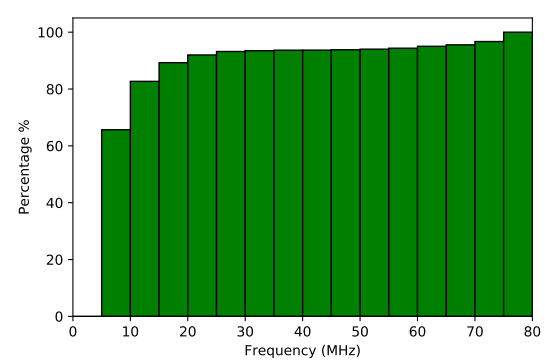

(a) O-QPSK (250 kbps)

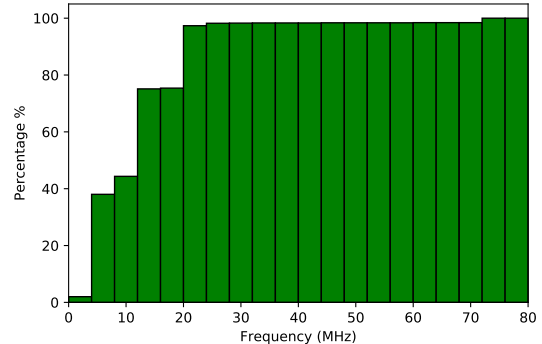

(b) OFDM option 1 MCS0 (100 kbps)

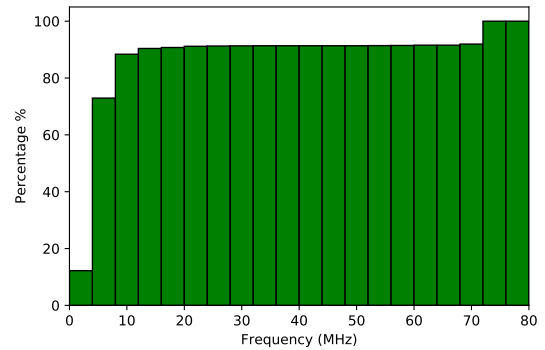

(c) OFDM option 1 MCS3 (800 kbps)

Figure 7: Coherence bandwidth.

it should be used. Our analysis is based on a $141,587,000-$ point connectivity dataset gathered indoors over 21 days.

We show that OFDM alone does not yield immunity to multi-path fading and external interference. We recommend combining OFDM with channel hopping at the MAC layer, with subsequent channels in the hopping sequence separated by at least $20 \mathrm{MHz}$. In the presence of nearby WiFi access points, we further recommend using OFDM option 1 MCS3 (i.e. without frequency repetition), with frames shorter than 127 B.

Since IEEE802.15.4 radios chips implementing multiPHYs are now available, as future work we perceive the need of a MAC layer that can dynamically configure the transceiver in order to choose the most convenient PHY according to desired range, latency, power consumption...for each pair of nodes.

\section{REFERENCES}

[1] 802.15.4-2015 - IEEE Standard for Low-Rate Wireless Networks, IEEE Std., April 2016.

[2] H. Khaleel, C. Pastrone, F. Penna, M. A. Spirito, and R. Garello, "Impact of Wi-Fi Traffic on the IEEE 802.15.4 Channels Occupation in Indoor Environments," in Conference on Electromagnetics in Advanced Applications, September 2009, pp. 1042-1045.

[3] W. Guo, W. Healy, and M. Zhou, "Impacts of 2.4-GHz ISM Band Interference on IEEE 802.15.4 Wireless Sensor Network Reliability in Buildings," IEEE Transactions on Instrumentation and Measurement, vol. 61, no. 9, pp. 25332544, 2012.

[4] T. Watteyne, C. Adjih, and X. Vilajosana, "Lessons Learned from Large-scale Dense IEEE802.15.4 Connectivity Traces," in Conference on Automation Science and Engineering (CASE), August 2015, pp. 145-150.

[5] C. Adjih, E. Baccelli, E. Fleury, G. Harter, N. Mitton, T. Noel, R. Pissard-Gibollet, F. Saint-Marcel, G. Schreiner, J. Vandaele, and T. Watteyne, "FIT IoT-LAB: A Large Scale Open Experimental IoT Testbed," in World Forum on Internet of Things (WF-IoT), December 2015, pp. 459-464.
[6] T. Watteyne, S. Lanzisera, A. Mehta, and K. S. J. Pister, "Mitigating Multipath Fading through Channel Hopping in Wireless Sensor Networks," in IEEE International Conference on Communications (ICC), May 2010, pp. 1-5.

[7] T. Watteyne, J. Weiss, L. Doherty, and J. Simon, "Industrial IEEE802.15.4e Networks: Performance and Trade-offs," in Conference on Communications (ICC). IEEE, June 2015, pp. 604-609.

[8] T. Watteyne, V. Handziski, X. Vilajosana, S. Duquennoy, O. Hahm, E. Baccelli, and A. Wolisz, "Industrial Wireless IPBased Cyber -Physical Systems," Proceedings of the IEEE, vol. 104, no. 5, pp. 1025-1038, May 2016.

[9] S. Lee, B. Kim, M. K. Oh, Y. Jeon, and S. Choi, "Implementation of IEEE 802.15.4g Wireless Communication Platform for Smart Utility Service," in Conference on Consumer Electronics (ICCE), Berlin, September 2013, pp. 287-289.

[10] J. Munoz, E. Riou, X. Vilajosana, P. Muhlethaler, and T. Watteyne, "Overview of IEEE802.15.4g OFDM and its Applicability to Smart Building Applications," in Wireless Days, 2018. 